\title{
Robotization as One of the Prospects for Electromobility in Belarus
}

\author{
Yankevich Natallia ${ }^{1, *}$ and Yankevich Stsiapan ${ }^{2}$ \\ ${ }^{1}$ Centre of the System Analysis and Strategic Research, the National Academy of Sciences of Belarus, \\ Republic of Belarus \\ ${ }^{2}$ OJSC "Instrument Making Plant OPTRON", the National Academy of Sciences of Belarus, Republic of \\ Belarus
}

\begin{abstract}
In order to create a competitive and resource-efficient transport system, the transport policy of the European Union provides for the achievement by 2030 of almost zero carbon dioxide content in the exhaust gases of vehicles used in large urban centers, and by 2050 the phasing out the use of cars, working on traditional fuels. The Republic of Belarus has a high scientific and sufficient industrial potential to participate in the process of promoting electric mobility, taking into account the use of robotics.

JSC "Instrument-Making Plant Optron" developed the working documentation and produced prototypes of typical representatives of the line of personal electric vehicles. However, the ongoing research focused on the creation of a preventive diagnostic system for the electric motorcycle, developed by Belarussian researches and its intelligent onboard system, focused primarily on real-time simulation processes, related specifically to the level of artificial intelligence, and on the implementation of executive level algorithms.
\end{abstract}

Keywords: Robotic electric cars, electric motorcycle, automated prediction of failure.

\section{INTRODUCTION}

One of the main tasks facing the European countries for the next few years is the creation of the most dynamically organized transport sector. The constant growth of passenger and freight traffic leads to congestion and pollution of transport highways and has a negative impact on people [1].

Belarus is sufficiently high car-equipped country in the Europe. By this indicator Belarus occupies an average position in the ranking of motorization (320 cars account for 1000 inhabitants) in the world. But the ecological situation in Belarus is also defined by the repercussions of the Chernobyl disaster. The analysis of the radioactive contamination of Europe by cesium137 shows that about $35 \%$ of the Chernobyl fallout of this radionuclide on the European continent is in Belarus. Pollutions on the territory of Belarus with cesium-137 with a density of over $37 \mathrm{kBq} / \mathrm{m}^{2}$ amounted to $23 \%$ of the total area of the Republic (for Ukraine $-5 \%$, Russia - $0.6 \%$ ). So, in such situation the negative influence of car pollutions on the health of Belarusians rises in times.

So electromobility is very important for Belarus. Moreover, it is expected excess of electricity in Belarus: Belarusian NPP now is building. Two blocks are being built with a total capacity of about $2,400 \mathrm{MW}$ - that is

Address correspondence to this article at the Centre of the System Analysis and Strategic Research, the National Academy of Sciences of Belarus, Republic of Belarus; E-mail: lab_12@tut.by additionally almost $50 \%$ of what Belarus consumes. Therefore, the issue of expenditure of electricity generated becomes very relevant and so the development of electric transport can if not solve this problem then at least make it less acute and essential.

At the moment, for the urban cycle, electric cars are the optimal type of passenger transport that meets all the requirements of the Belarusian environmental and energy security. At the same time, during a number of the conducted sociological polls, it was revealed that $65 \%$ of respondents do not plan to buy an electric car even with "zero" customs clearance, as long as the necessary infrastructure and services for them are absent in Belarus. $18 \%$ of respondents are categorically and will not use the electric cars until the cars with ICE have been producing. $12 \%$ of users believe that one needs to use the moment and buy an electric car right now - as long as there are customs indulgences. And, unfortunately, only $5 \%$ of respondents are sure that electric motors are the future and they are ready to buy electric cars even if they have to pay customs duties. The majority of respondents $(61.2 \%$ of respondents) would hardly have ventured to buy an electric car until there is an appropriate infrastructure for them. However, according to statistics in the world, $90 \%$ of drivers travel no more than $90 \mathrm{~km}$ daily. All this makes the development of the personal light electric transport segment (electric scooters, electric bicycles, electric motorcycles), which are an excellent alternative to an electric car, relevant.

At the same time, the need of introduction of new technologies, including robotic approaches, actualizes 

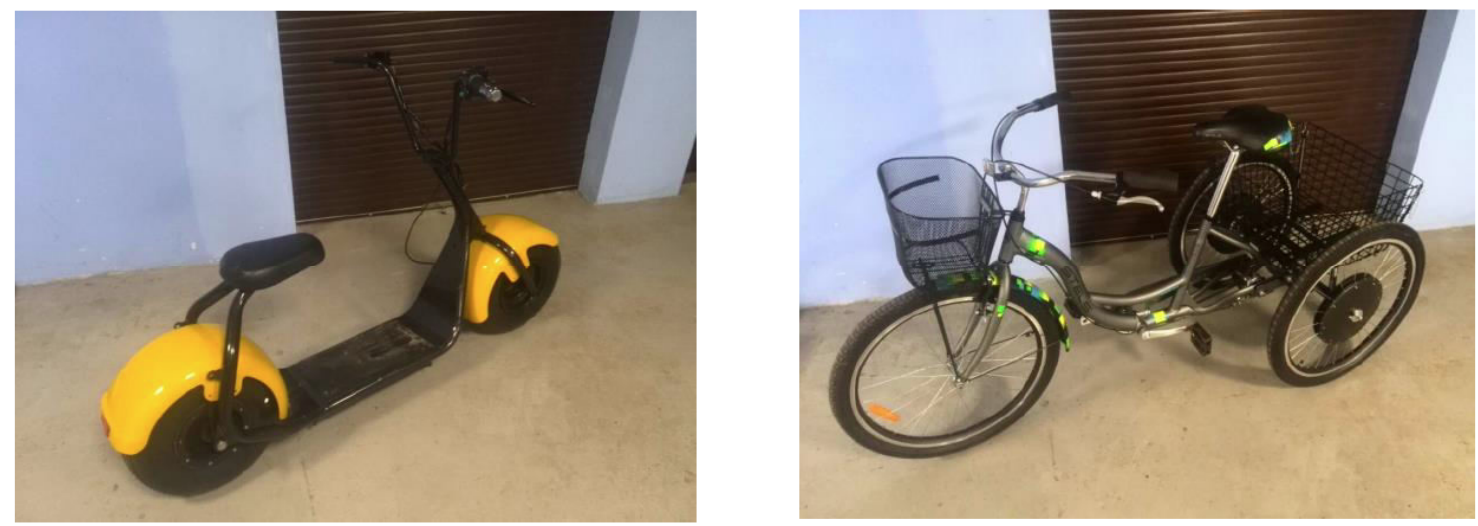

Figure 1: Prototype of electric scooter and three-wheeled electric bicycle, developed by OJSC "Instrument Making Plant OPTRON".

the usage of modern approaches in the development of personal light electric transport.

OJSC "Instrument Making Plant OPTRON" had developed working documentation. So the prototypes of typical representatives of personal electric vehicles were produced: a motor kit for converting a bicycle to electric traction, a city folding electric bicycle, a threewheeled electric bicycle, electric scooters based on motor wheels with power up to $1000 \mathrm{~W}$ (Figure 1).

Series of motor-wheels have been developed on the base of composite materials from nano-modified iron powder. Usage of such materials in the components of the electric motors makes it possible to increase the efficiency of the products by $10-20 \%$ due to reducing the eddy current loses and substantially reducing the production costs.

The design of the proposed motor-wheels provides for a compact arrangement the engine and supplying batteries inside of them.

Motor wheels are designed for power 36-volt lithium-ion battery $3500 \mathrm{mAh}$.

An intelligent control algorithm for a two-wheeled electric bike has been developed with the function of assisting pedaling, smooth deceleration and recovery, as well as a controller for a three-wheeled electric bike with a two-wheel drive, which implements differential control of the linear speed of rotation of motor-wheels during rotation.

Based on these studies, the issue of expanding the range of personal electric vehicles that meet the requirements of potential customers and various end users (urban and rural residents, state and commercial structures, rental services) by the best way has been worked out: urban electric scooter, three-wheeled cargo electric scooter and electric motorcycle.

At the same time, work is underway to create an "intelligent" motorcycle (Figure 2).

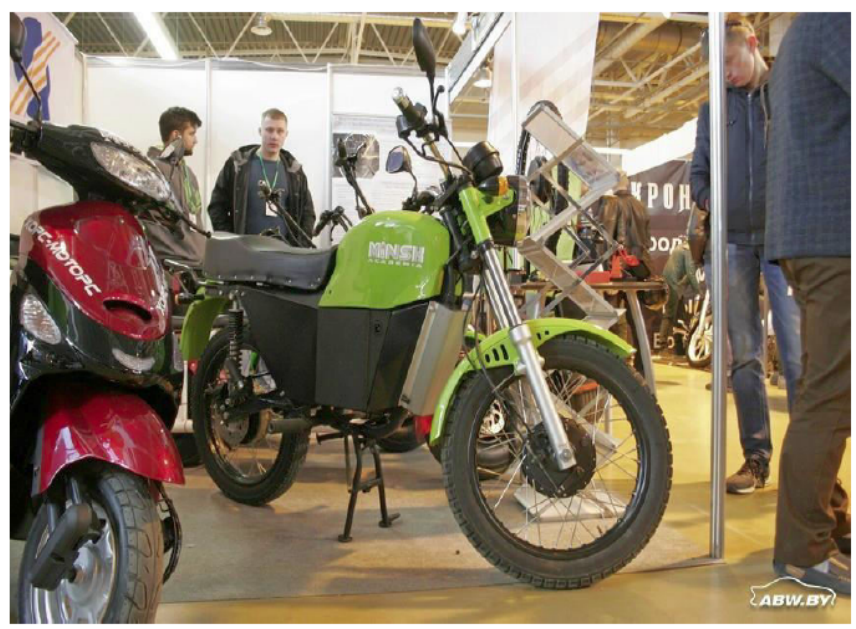

Figure 2: Electric motorcycle, developed by OJSC "Instrument Making Plant OPTRON".

It is known that the use of automated vehicles helps to solve the following problems:

- to free a person from the task of control in routine situations, for example, during prolonged traffic on highways, in traffic jams, and in the urban traffic;

to reduce the accident rate, a significant proportion of which is associated with the socalled "human factor". The use of "intelligent" automated vehicles can significantly reduce the number of road accidents, as $80 \%$ of accidents are caused by the human factor. Perhaps this is an overly optimistic forecast, since autopilot robots will make their mistakes typical of robots; 
- $\quad$ to improve the economy of a vehicle using - due to energy economy;

- $\quad$ to save on the creation of road infrastructure. For example, robot-drivers hardly need in dividing strips and barriers. The markup can be virtual for example, in cards in the autopilot's memory.

However, the ongoing research focused on the creation of a preventive diagnostic system for an electric motorcycle.

\section{State of the Question}

Currently, there are many technical means and methods for converting data into information used in the decision-making process. The development of the mathematical apparatus took place in parallel with the evolution of relevant scientific disciplines, such as statistics, operations research, and computer technologies. Computer programs make modern computational methods and complex prognostic models widely available.

Primary research or primary data analysis is an important stage in most statistical studies, not only in terms of their study and generalization, but also in the construction of simple models. The advantage of such analysis is its intuitive understandability, valuable for interpreting obvious trends in the development of phenomena. The most popular methods in the primary analysis are the one-dimensional methods that process each of the variables independently. Variables here mean time series of indicators of each individual event. Examples of such methods are histograms, tree diagrams, and diagrams in which the connections between elements are indicated by arrows. These graphical methods provide visual information about the data and are a widely used research tool in practice [24].

The purpose of the analysis of time's series is to identify the main factors affecting the dynamics of an event using statistical methods. Sometimes such an analysis allows identifying the most obvious trends of the event, however, if repeatability or uniform cycles are observed in the data series, its application can be associated with serious difficulties. In this case, charts or diagrams combining performance data contain conflicting information. They reflect only surface features that are noticeable obviously, the causes of which are hidden in a variety of manifestations. The effectiveness of the time series analysis method is highly dependent on the quality of the source information.

The analysis of the state of the event is the development of the method of temporary analysis of individual indicators. In this case various coefficients that are obtained by certain algebraic formulas from simple indicators are analyzed instead of individual indicators, often using weight coefficients determined by experts. A large number of different methods for calculating such coefficients are known, some of them are based on the assumption of the existence of one complex indicator that best reflects the state of the event. Typically, such approaches are used with the aim of one-dimensional ordering of various events by the value of a complex indicator designed to serve as a measure of comparison. However, all of them are not free from the subjectivity of the authors of the methods. Despite the fact that such approaches have been developed for more than a hundred years, the hypothesis of the existence of a single universal methodology has not yet been proved before now [5].

In general, the method of one-dimensional ordering has a significant drawback. Being intuitively attractive, one-parameter descriptions are too approximate for optimal decisions to be made based on them.

The use of neural networks for data processing is connected with a number of difficulties. In some cases, the input data are correlated with the desired output data or can be characterized using a number of persistent factors. This is tantamount to the a priori assumption of the existence of certain rules governing the transformation of the original time series into a set of output data. All this leads to the problem of identifying representative series and factors that are significant in terms of the characteristics of the input data, as well as significant examples. The complexity of using adaptive neural networks in this case is due to the fact that the dimension of the output parameters plane must be determined before training begins. In addition, in the process of self-training of a neural network, the addition of new neurons is not allowed. Thus, the adaptive neural network method is sometimes not flexible enough [6].

It is advisable to supplement the adaptive neural network method with genetic algorithms in such cases. A hybrid neural network model that combines the training procedure of the adaptive neural network method with an evolutionary model significantly increases the flexibility of used neural structure. A 
hybrid model can provide more complete and meaningful information about the processes under study (both qualitative and quantitative).

In general, the use of an adaptive neural network does not imply a complete rejection of other well-known methods, and it is advisable to supplement network models with other analysis methods. It is believed that the results obtained using adaptive neural networks can often be improved if they are used in combination with traditional statistical methods or in combination with other progressive tools, such as multidimensional statistical models; trained neural networks; genetic algorithms, fuzzy logic. Each of the additional methods most closely meets the solution of some special aspects of the tasks being solved. Therefore, for each of these aspects, the most appropriate methods should be chosen. The most promising approach in the use of adaptive neural networks is the integration of this method into an integrated decision support information system.

The concept of the degree of separation plays a special role in fuzzy choice models. It is used when choosing an alternative in fuzzy choice models. The difference in degree of separation reflects the difference in degree of clarity between statements about the choice of alternatives.

The problem of distinguishing between alternatives or objects with a degree of fuzziness raises two questions in decision theory:

1. If the degree of separation is low, then the solution leads to the need to collect more information. The final decision is made when the degree of separation exceeds a certain level.

2. The formulated procedural recommendation is made under the assumption that the degree of distinction will increase after selecting additional information or at least not decrease [7].

Namely such formulation raised the question of the applicability of the theory of fuzzy sets to the study of the reliability of parts of technical systems $[6,7]$. This is especially true when building complex models that take into account the personality factor (the model "a man the external environment - a technical system"), which requires the use of semantic approaches in the development of analytic methods.

Systematic approach has the particular attention in the study of vehicle reliability. Despite the fact that the systematic approach is widely used in solving a number of issues, the conceptual basis for its application in considering reliability problems has not yet been formulated. At the same time, the presence of clear provisions defining the legitimacy of certain decisions will significantly increase the effectiveness of the method, not only focusing on the most important aspects, but also taking into account the most significant limitations to the maximum possible extent.

But in practice it is not possible in most cases to organize tests for obtaining experimental data on the reliability of the required type and in sufficient volume. Usually the task is to evaluate the reliability indicators for the statistical material that is available. At the same time, the nature of the statistical material is significantly affected by the test strategy (or operating mode), namely: the number of samples subjected to tests, the procedure for monitoring the functioning in the process of testing (observation), procedure for restoration (replacement) of products, order of receipt of parts for testing, criterion for the completion of tests (observations). Actually listed factors can vary significantly depending on specific conditions, for example: testing a single product or group of products, continuous or periodic monitoring only before or at the end of tests [8].

Various combinations of these factors cause a wide variety of real test strategies in practice. However, in reality, the choice of strategy is largely determined precisely by the spectrum of the loads acting on the part. At the same time, it is sometimes difficult to determine the loads that have the most significant effect on the occurrence of a failure. First of all, this is due to the fact that a number of loads (for example, environmental impact) are random in nature.

From the point of view of the nature of a priori information about the distribution function, the whole variety of practical problems is reduced essentially to the following two options:

1. The form of the distribution function of the observed random variable is known a priori. The task of statistical processing is to obtain estimates for reliability indicators, taking into account the type of distribution function and the nature of the changing statistical material.

2. The form of the distribution function of the observed random variable is unknown or known only tentatively. In this case, based on the 
analysis of the processes leading to failures, the operating experience of similar products, and a preliminary analysis of the information obtained during the tests (for example, by the type of histogram), a certain hypothesis about the form of the distribution function is accepted. The task of processing is to check whether the experimental data do not contradict the accepted hypothesis and to evaluate the parameters of this distribution function.

Significant progress in carrying out such studies has been achieved with the development of theories of fuzzy sets and possibilities. But the statement of the problem from the point of view of probability theory assumes that the nature of the considered phenomena and the corresponding probability distribution are known. Uncertainty implies that the possible states of the phenomenon are known, but the probability distributions are not defined. Currently, the main discussion is around modeling and processing uncertainty between adherents of fuzzy-multiple procedures and other non-standard approaches and advocates of the use of classical probabilistic and statistical methods. So, the simplest argument has been put forward in favor of probability theory instead of the theory of fuzzy sets, namely that the distributions of a priori and conditional probabilities are enough to describe all the problems of uncertainty (under the often impossible condition in practice that they can be obtained) [6].

One of the most important areas of scientific research in the development of promising vehicles is the introduction of new technologies, the use of which can ensure the creation of a vehicle with artificial intelligence. In the field of technical diagnostics, the essence of using the elements of an artificial approach is to help human actions in the planning, implementation and processing of diagnostic research results, especially for complex technical objects.

This trend also affects electric vehicles to a large extent. Therefore, the diagnostic systems being developed today, the operation of which is more or less connected with the artificial intelligence of the machine (neural network), which allow real-time monitoring (control) of the flow of goods, are the first significant step in the direction of creating autonomous "intelligent" vehicles.

The "intelligent" on-board system of a vehicle is focused primarily on real-time simulation processes related specifically to the level of artificial intelligence, and on the implementation of executive-level algorithms. In fact, intelligent control and management systems are only the development of traditional control systems, adding some additional functions with a known set of methods for their implementation. So, in "intelligent", as well as conventional control systems, the same sources of information are used: sensors for forming a model of the external environment, receiving external special information and behavior (state of the operator). The only new thing is that the number and purpose of these sensors is increasing, and the information (naturally, appropriately processed) does not reach a driver: a significant part of it goes to the executive elements of the systems of an "intelligent" vehicle.

Intelligent management architecture has the following levels typically:

- operational;

- functional subsystems;

- $\quad$ sensors;

- $\quad$ executive bodies;

- necessary information links with the external environment.

Architectural principles determine the levels and nature of inter-level information links between general modules within the system, as well as with the external environment. For a vehicle, communications of three levels are best applicable: local information level (i.e. between sensors and signal processing modules, consistent with their characteristics and placement), high-speed intermodular and functional devices level within a single on-board computer system.

As a rule, modeling proceeds from two principles for implementing intelligent control of human-machine systems. The first one of them is that the environment, driver and subsystems of the vehicle can be represented as a system of observers of varying degrees of completeness. Moreover, if the functional extremely dynamic subsystems of an object should be constructed as sufficiently complete, then all others should be described extremely simplistically. On the other hand, their mathematical description should be as accurate as possible in order to predict their condition, including gradual and sudden failures. As for the driver, given the high parametric variability of the state of a 
person, his model should be extremely simplified and basically identify the possibility of an unfavorable forecast for the development of the surrounding world and the position of the object in it [9].

Intelligent systems solve problems requiring increased reliability (motion control, brake systems, alarm systems), as well as increased speed (processing of external signals) in the conditions of fuzzy formalization of initial conditions. In particular, it is important to carry out modeling processes related to the level of artificial intelligence in real time, to take into account environmental changes and, in addition, to correct erroneous actions of the driver.

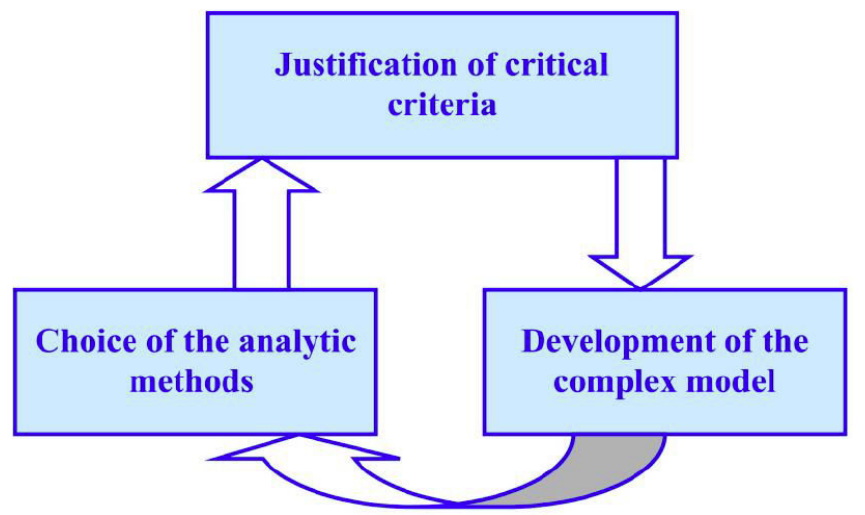

Figure 3: Decision making process in the analysis of complex technical systems.

It is obvious that the approaches described above and used in the analysis of the reliability of complex systems naturally fit into the principles of construction and can be successfully used in the development of intelligent vehicle systems. When wondering about the possibilities of processing information received from the diagnostic system, one can concentrate on a simple statement of the fact of a malfunction (sensor readings indicating a deviation or dangerous proximity of a controlled parameter to a given dangerous limit), and a subsequent analysis of the possible consequences of this deviation using a neural network. The decisionmaking process can be reduced to the following sequence of actions (Figure 3 ):

- selection of critical criteria and verification of their consistency;

- development of a structural (or graph) model of a complex system (selection of elements, assignment of relationships);

- $\quad$ selection (or development) of effective methods for analyzing the resulting model.

As the result of carrying out of this sequence of actions, the criteria can be modified so that the cycle appears (Figure 3 ).

Numerous studies have established common approaches and limitations used in predicting the reliability of complex systems.

In general, this process can be represented algorithmically as a sequence of actions (situations), shown in Figure 4.

Thus, the algorithmic construction of a neural network for failure analysis is leading to the development of an information state graph according to the methodology described in [1]. The analysis should be based on a failure database generated for specific vehicle models. The number of failures (statistical information by years, probabilistic calculation) can be presented either as a data set or obtained as a combination of known distributions.

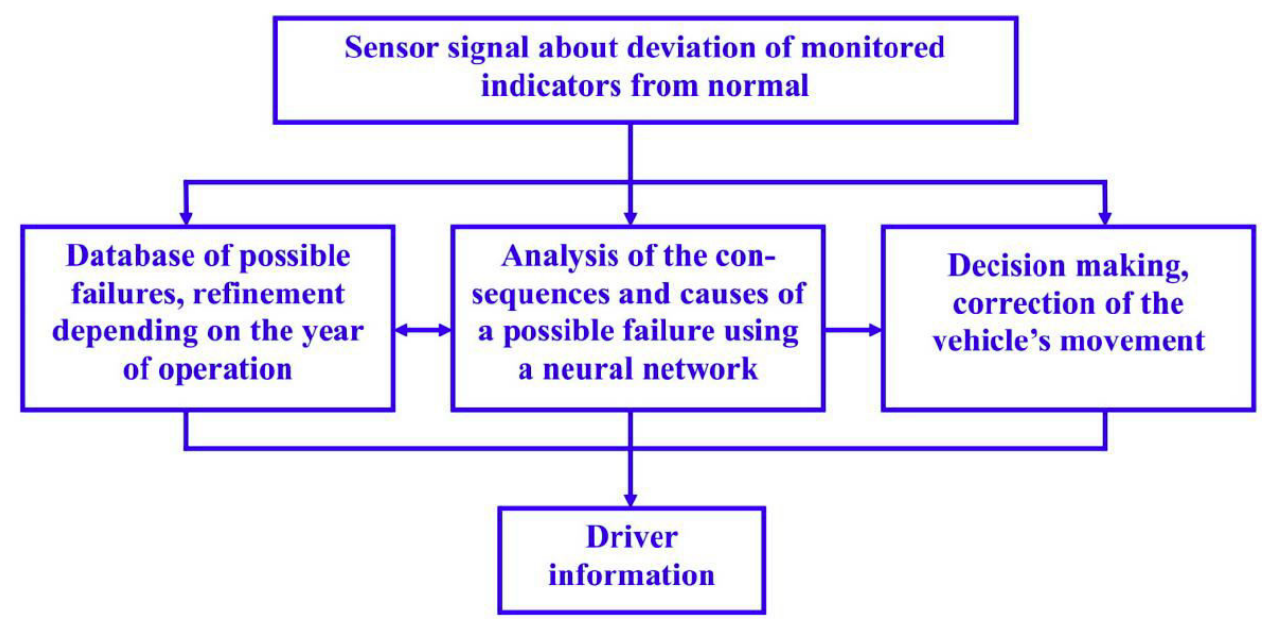

Figure 4: Artificial Intelligence System for Vehicle Failure Analysis. 


\section{Obtained Results}

The study was carried out in the following areas:

- $\quad$ study of failures of elements of a complex technical system and the causes of their occurrence;

- establishing links between elements of a complex technical system;

- development of methods for the analysis of complex technical systems both globally and locally;

- study of controversial (for example, from the point of view of manufacturability) recommendations received and the achievement of a compromise in the refinement of the general model.

The mathematical approach in this case, generally speaking, was used in two ways - both for formulating and solving the general staging part of the analysis of the reliability of complex systems, and for solving local issues (for example, analysis of the stress state of the elements). It can be stated that the mathematical formulation of the problem of studying the reliability of a complex system already carries information about possible outcomes and the choice of the optimal strategy. At the same time, perhaps, one should not exaggerate the role of mathematical theories in achieving a result. The strength of these approaches lies in developing a search strategy that can lead to a result. So, counting the chances in any gambling game does not provide any gain or loss, it only contributes to the choice of a strategy for behavior in games. At the same time, the player understands the situation, but cannot change it in his favor in each particular game. However, this kind of information can be of great benefit in choosing a strategy.

Moreover, the explanation of the behavior of the system and its states is primarily associated with the need to clarify what properties, to what extent and under what conditions this system has. It is believed that at the first stage, the structure and properties of the system are refined using ensembles of different by their organization models. This way of concretizing the abstract system is effective in case of difficulties in clarifying the knowledge about the properties of the system with the help of detailing the special structures representing these properties, and can be implemented by constructing a hierarchical structural model, which, with all its clarity, does not carry enough information to describe the reliability of operation system.

The proposed algorithm was implemented in the development of the diagnostic system of an electric motorcycle, which performs the following functions:

1) storage of the "technical passport" of the motorcycle (state vehicle number, vehicle model, chassis number, engine number);

2) registration of engine start (with reference to time);

3) calculation of the total engine operating time (in hours);

4) interrogation of the voltage sensor of the onboard network and registration of the parameter output beyond the permissible limits in nonvolatile memory (with reference to time);

5) interrogation of the engine speed sensor and registration of the parameter output beyond the permissible limits in non-volatile memory (with reference to time);

6) continuous registration (period from 5 to 60 seconds), pre-selected parameters (voltage onboard network) with recording in non-volatile memory;

7) interrogation of the ambient temperature sensor;

8) transmitting data on the state of sensors to external devices (upon receipt of a request);

9) transfer of data on the exit of temperatures or pressures beyond the permissible limits to the interface unit;

10) transfer of statistical data to a $P C$ upon request from the latter.

As a result of the analysis of the functions performed by the system, and based on the modular structure, the system was divided into hardware and software modules. The result of the partition in the form of a modular structure of the system is presented in Figure 5.

In accordance with the structural diagram, a circuit is developed for the electrical circuitry and the electronic board of the engine diagnostic system. 


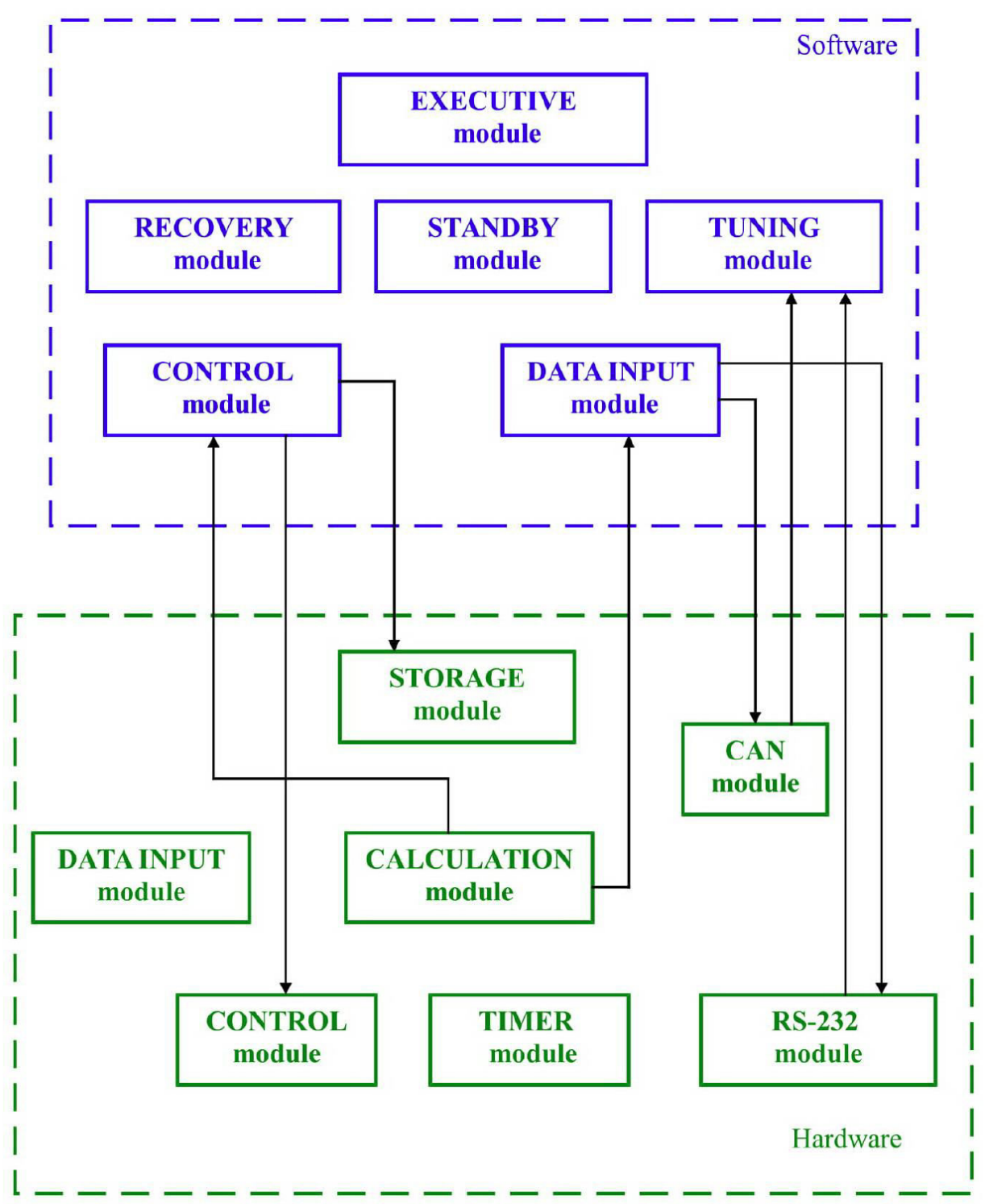

Figure 5: Interconnections between hardware and software diagnostic system of an electric motorcycle.

\section{CONCLUSIONS}

The proposed approach can be presented as an independent neural network, or as part of a more global information system.

It is supposed, that the decision-making process can be reduced to the following sequence of actions:

- $\quad$ selection of critical criteria and verification of their consistency;

- development of a structural (or graph) model of a complex system (selection of elements, assignment of relationships);

- $\quad$ selection (or development) of effective methods for analyzing the resulting model.
The algorithmic construction of the proposed neural network for failure analysis is based on a failure database generated for specific vehicle models. The number of failures (statistical information by years, probabilistic calculation) has been presented as a combination of known distributions.

In any case, usefulness of such approach is obvious: such system allows implementation early diagnostics in real time, to predict both the consequences of a failure, and its causes, as well as adjust the movement of the vehicle.

Nevertheless, the exploitation of the proposed model is of a long-term objective due to lack of realtime data on reliability of the motorcycle and necessity of approbation of proposed solutions in real conditions. 
In any case, its usefulness is obvious: such system allows implementation early diagnostics in real time, to predict both the consequences of a failure, and its causes, as well as adjust the movement of the vehicle.

\section{REFERENCES}

[1] Yankevich N. Adaptation of Graph and Game Theories to Reliability Problems. In Garbolino E, Yankevich N, et al. (Eds.), Transport of Dangerous Goods, NATO Science for Peace and Security Series C: Environmental Security, Springer Science + Business Media B.V. 2012; 167-207. https://doi.org/10.1007/978-94-007-2684-0 7

[2] Bolotin VV, Gusenkov AP, Nefedov SV, Tananov Al. Reliability in technics. Scientific, technical, economic and legal aspects of reliability. Bolotin VVM, Ed. MNTK Reliability of machines 1993; p. 252.

[3] Bolotin VV, Nefedov SF, Chirkov VP. et al. Reliability in technology. Methodology of calculated forecasting of reliability indicators. Methods of probability theory. Methodological manual. Scientific and technical publication NTP-3-93. Bolotin VVM, Ed. MNTK Reliability of machines 1993; p. 172.
[4]

Bolotin VV, Kovekh VM, Nefedov SP, Tananov Al, et al. Reliability in technology. Reliability Performance Prediction Methods. A systematic approach/ Methodological manual. Scientific and technical publication NTP-4-96. Ed. Bolotin V.V. M .: MNTK "Reliability of machines 1996; p. 217.

[5] Reinschke K. Models of reliability and sensitivity of systems. M .: Mir, 1979.- 450 p. https://www.studmed.ru/raynshke-kmodeli-nadezhnosti-i-chuvstvitelnostisistem_3636f04618b.html

[6] Fuzzy sets and the theory of possibilities. Recent Achievements / Ed. R.R. Yager. - $M$.: Radio and communications, 1986. - 408 p. http://en.bookfi.net/book/ 479837

[7] Ackoff, R. The Art of Problem Solving. M .: Mir, 1982.- 324 p.

[8] Reliability of technical systems: Reference book. Belyaev YuK, Bogatyrev VA, Bolotin VV, and etc.; Ed. Ushakova I.A. M .: Radio and communications 1985; p. 608.

[9] Resch B, Mittlboeck M, Girardin F, Britter R, Ratti C. Live Geography - Embedded Sensing for Standardised Urban Environmental Monitoring. International Journal on Advances in Systems and Measurements 2009; 2(2\&3): 156-167.

DOI: https://doi.org/10.31875/2409-9694.2019.06.3

\section{(C) 2019 Natallia and Stsiapan; Zeal Press}

This is an open access article licensed under the terms of the Creative Commons Attribution Non-Commercial License (http://creativecommons.org/licenses/by-nc/3.0/) which permits unrestricted, non-commercial use, distribution and reproduction in any medium, provided the work is properly cited. 